\title{
MODELAGEM DA DISTRIBUIÇÃO DIAMÉTRICA DE UM FRAGMENTO DE FLORESTA OMBRÓFILA MISTA EM LEBON RÉGIS, SC
}

\author{
Saulo Jorge Téo ${ }^{1}$, Felipe Marcon ${ }^{2}$, Chaiane Rodrigues Schneider ${ }^{2}$, Francieli Baggio dos Santos ${ }^{2}$, \\ Karine Maria Atuatti Chiarello ${ }^{2}$, Luan Demarco Fiorentin ${ }^{2}$ \\ ${ }^{1}$ Universidade do Oeste de Santa Catarina, Departamento de Engenharia Florestal, Xanxerê, Santa Catarina, Brasil - \\ sauloteo@yahoo.com.br \\ ${ }^{2}$ Universidade do Oeste de Santa Catarina, Curso de Graduação em Engenharia Florestal, Xanxerê, Santa Catarina, Brasil - \\ felipemarconnn@hotmail.com; chai.rodriguesschneider@gmail.com; francieliefrancieli@yahoo.com.br; \\ karinechiarello@uol.com.br; luanfiorentin@hotmail.com
}

Recebido para publicação: 13/12/2013 - Aceito para publicação: 30/09/2014

\begin{abstract}
Resumo
O objetivo deste trabalho foi ajustar e selecionar diferentes funções de densidade de probabilidades (FDP), tais como Normal, Ln-normal, Gamma (Adaptada), Beta (Adaptada), Exponencial, Weibull 3P, Sb de Johnson, Weber e Péllico Netto, para descrever a distribuição diamétrica de todas as espécies e das sete espécies com maior valor de importância de um fragmento de Floresta Ombrófila Mista em Lebon Régis, SC. O processamento dos dados foi realizado por meio da ferramenta "Solver" MS Excel 2007, a qual utiliza o algoritmo linear de gradiente reduzido generalizado (GRG) na interação dos parâmetros. Os critérios de seleção utilizados foram o teste de Kolmogorov-Smirnov e as estatísticas de ajuste e precisão. De acordo com os resultados, a função que melhor representou a distribuição diamétrica considerando todas as espécies do fragmento florestal foi a Sb de Johnson. Quando consideradas as sete espécies individualmente, a função Ln-normal melhor descreveu a distribuição diamétrica de Myrcia sp. e Myrsine gardneriana (Aubl.) DC. Já para Ocotea porosa (Nees \& C. Mart.) Barroso e Araucaria angustifolia (Bertol.) Kuntze, a função de melhor aderência foi Gamma (Adaptada), e para Ocotea elegans Mez, Mimosa scabrella Benth. e Styrax leprosus Hook. \& Arn., as funções de melhor desempenho foram Weibull 3P, Péllico Netto e Normal, respectivamente.

Palavras-chave: Floresta com Araucária; estrutura horizontal; função de densidade de probabilidade.
\end{abstract}

\begin{abstract}
Modeling the diameter distribution of a fragment of Mixed Ombrophyllous Forest, in Lebon Régis, SC. The objective of this work was to fit and to select different probability density functions (pdf's), such as, Normal, Gamma (Adapted), Beta (Adapted), Exponential, Weibull 3P, Johnson Sb, Weber and Péllico Netto, to describe the diameter distribution of all species and the seven species with the highest importance value of a fragment of Mixed Ombrophyllous Forest, in Lebon Régis, SC, Brazil. The data processing was performed using the Solver tool on MS Excel 2007, using the linear algorithm of generalized reduced gradient (GRG) for interaction of parameters. The selection criteria used were the Kolmogorov-Smirnov and statistics of fit and accuracy. According to the results, the function that best represented the diameter distribution considering all species of forest fragment was Johnson $\mathrm{Sb}$. When considered the species individually, the Ln-normal function best described the diameter distribution of Myrcia sp., and Myrsine gardneriana (Aubl.) DC. For Ocotea porosa (Nees \& C. Mart.) Barroso., and Araucaria angustifolia (Bertol.) Kuntze., the function with the best adherence was Gamma (Adapted) and Ocotea elegans Mez., Mimosa scabrella Benth., and Styrax Leprosus Hook. \& Arn., functions with the best performance were, 3P Weibull, Péllico Netto and Normal, respectively.

Keywords: Araucaria Forest; horizontal structure; probability density function.
\end{abstract}

\section{INTRODUÇÃO}

A Floresta Ombrófila Mista, também conhecida como Floresta com Araucária, representa uma das formações vegetais, por ser uma rica mistura florística, a qual comporta floras de diferentes origens, a tropical afro-brasileira e a temperada austro-brasileira, inseridas na área de domínio da Mata Atlântica,

FLORESTA, Curitiba, PR, v. 45, n. 2, p. 337 - 348, abr. / jun. 2015.

Téo, S. J. et al.

ISSN eletrônico 1982-4688 / ISSN impresso 0015-3826 
típica da região Sul do Brasil, abrigando uma ampla variedade de espécies, algumas das quais só localizadas nesse ecossistema (CAPOBIANCO, 2002; CAMPANILI; SCHAFFER, 2010).

De acordo com Schaaf et al. (2006) e Machado et al. (2009b), nota-se uma crescente apreensão por parte dos pesquisadores e da sociedade em geral, uma vez que o desenvolvimento sustentável é de fundamental importância no processo do desenvolvimento socioeconômico com a conservação da natureza, em que restaurar, conservar e aproveitar prudentemente as benfeitorias sucedidas da Floresta Ombrófila Mista compõem um grande desafio.

Entre as várias maneiras de conhecer a estrutura da floresta para auxiliar em sua correta preservação ou conservação destaca-se o estudo da distribuição diamétrica, uma vez que ela é uma forte indicadora da estrutura florestal, servindo para distinguir tipologias florestais e fornecer base para identificar a intensidade da regeneração natural em grau de espécie, bem como para a floresta como um todo, além de ser uma extraordinária medida do estoque em crescimento (SCOLFORO, 2006).

Arce (2004) e Loetsch et al. (1973), afirmaram que a distribuição diamétrica é a ferramenta mais simples e poderosa para caracterizar a estrutura de uma floresta, permite elaborar conclusões a respeito da estrutura da floresta. De um modo geral, o diâmetro se correlaciona muito bem com outras variáveis importantes como altura, volume e sortimentos florestais ou classes de utilização da madeira.

Para Clutter et al. (1983) e Machado et al. (2009a), a análise da distribuição diamétrica possibilita estimar o número de árvores por hectare, para cada classe de diâmetro. Além disso, pode gerar a altura média nas classes de diâmetro, permitindo a descrição mais detalhada da estrutura de produção do povoamento, sendo uma importante ferramenta na avaliação da dinâmica de florestas nativas e plantadas.

Entre os tipos de distribuição diamétrica de florestas, podem-se citar a distribuição unimodal, a distribuição decrescente e a distribuição multimodal (LOETSCH et al., 1973; SCOLFORO, 2006). A distribuição unimodal é característica de povoamentos florestais jovens e equiâneos. A distribuição decrescente, também chamada de J-invertido, é caracterizada pela diminuição do número de árvores por unidade de área à medida que o diâmetro aumenta, ocorre principalmente em florestas nativas. E a distribuição multimodal apresenta mais de um ponto de maior frequência, podendo ser resultado de espécies de diferentes desempenhos ou idades, diferentes qualidades de sítio na floresta, ou ainda resultado de tratos silviculturais.

A melhor forma de descrever a estrutura diamétrica de uma floresta ou de uma espécie é através da aplicação de funções de densidade probabilísticas (FDP), cujas distribuiçõos admitem obter a probabilidade de as árvores incidirem dentro de classes de diâmetro em que haja um limite inferior e outro superior. Vale ressaltar que, devido às características de cada espécie florestal, surge a necessidade de que as FDP utilizadas para expor a estrutura diamétrica sejam devidamente testadas e selecionadas, de forma a identificar qual delas proporciona maior consistência para descrever o comportamento da variável (BARTOSZECK et al., 2004; SCOLFORO, 2006; MACHADO et al., 2009a; MACHADO et al., 2009b; MACHADO et al., 2010a).

Dessa forma, o objetivo deste estudo foi ajustar diferentes FDP para descrever a distribuição diamétrica de um fragmento de Floresta Ombrófila Mista em Lebon Régis, SC, bem como avaliar e selecionar a FDP de melhor aderência aos dados da distribuição diamétrica observada nas espécies florestais estudadas, identificando o tipo de distribuição diamétrica para diferentes espécies de maior valor de importância.

\section{MATERIAL E MÉTODOS}

A área de estudo está localizada no município de Lebon Régis, Santa Catarina, na Fazenda São Sebastião, com 392,95 hectares de extensão, situada na região centro-oeste do estado, com latitude de 26 56'07' e longitude de 5041'17', em áreas da empresa Juliana Florestal Ltda., de Caçador, SC.

De acordo com Caldato et al. (1999), o clima é mesotérmico, subtropical úmido, sem estação seca, com verões frescos. Segundo a classificação de Köppen, é uma região de ocorrência de clima tipo $\mathrm{Cfb}$, isto é, temperado úmido, com geadas severas e temperatura média de $15,8^{\circ} \mathrm{C}$.

A vegetação original da região de estudo é a Floresta Ombrófila Mista, principalmente em sua formação Floresta Ombrófila Mista Montana, a qual ocupava quase inteiramente o planalto acima de 500 m de altitude, nos estados do Paraná, Santa Catarina e Rio Grande do Sul (IBGE, 2012).

Os principais solos da área de estudo são Cambissolos háplicos, com grande variação de profundidade e drenagem variando de acentuada a imperfeita, e Nitossolos brunos, de textura argilosa ou muito argilosa, em geral moderadamente ácidos a ácidos (IBGE, 2007). 
Os dados para a realização desta pesquisa foram obtidos por amostragem aleatória simples. As 20 parcelas amostradas foram feitas utilizando-se o método de área fixa, com dimensões $10 \mathrm{x} 50 \mathrm{~m}$ $\left(500 \mathrm{~m}^{2}\right)$. Em cada parcela foram medidas as árvores que apresentavam diâmetro à altura do peito (d) $\geq 5 \mathrm{~cm}$.

Foram amostrados 1703 indivíduos, a fim de se avaliar quais espécies apresentaram maior valor de importância na área de estudo, uma vez que, por meio desse critério, 7 espécies foram selecionadas para descrever a distribuição diamétrica do fragmento de Floresta Ombrófila Mista: Myrcia sp., Ocotea porosa (Nees \& C. Mart.) Barroso, Araucaria angustifolia (Bertol.) Kuntze, Ocotea elegans Mez, Myrsine gardneriana (Aubl.) DC., Mimosa scabrella Benth. e Styrax leprosus Hook. \& Arn. Além de estudar separadamente a distribuição diamétrica das 7 espécies, conforme descrito anteriormente, também foram ajustadas e testadas as diferentes FDP, considerando-se todas as espécies do fragmento florestal.

Para todas as espécies e para cada espécie estudada, foram ajustadas e testadas as FDP Normal, Ln-normal, Gamma (Adaptada), Beta (Adaptada), Exponencial, Weibull 3P, Sb de Johnson, Weber e Péllico Netto (Tabela 1).

Tabela 1. Funções de densidade probabilísticas ajustadas para descrever a distribuição diamétrica para todas as espécies e para as sete espécies com maior valor de importância em um fragmento de Floresta Ombrófila Mista.

Table 1. Probability density functions fitted to describe the diameter distribution for all species and the seven species with the highest value of importance in a fragment of Mixed Ombrophyllous Forest.

\begin{tabular}{|c|c|c|}
\hline Nome & FDP & Condições \\
\hline Normal & $f(x)=\frac{1}{\sigma * \sqrt{2 \pi}} * e^{\left\{-\frac{1}{2} *\left[\frac{(x-\mu)^{2}}{\sigma^{2}}\right]\right\}}$ & $\begin{array}{c}\sigma>0 \\
-\infty<x<+\infty \\
-\infty<\mu<+\infty\end{array}$ \\
\hline Ln-normal & $f(x)=\frac{1}{\sigma * \sqrt{2 \pi}} * e^{\left\{-\frac{1}{2} *\left[\frac{(\ln x-\mu)^{2}}{\sigma^{2}}\right]\right\}}$ & $\begin{array}{c}x \geq x_{\min } \\
\sigma<0 \\
-\infty<x<+\infty \\
-\infty<\mu<+\infty\end{array}$ \\
\hline $\begin{array}{l}\text { Gamma } \\
\text { (Adaptada) }\end{array}$ & $f(x)=\frac{\left(x-x_{\min }\right)^{(\alpha-1)} * e^{-\left[\frac{\left(x-x_{\min }\right)}{\beta}\right]}}{\gamma}$ & $\begin{array}{c}x \geq x_{\min } \\
\alpha, \beta, \gamma>0 \\
-\infty<x_{\text {min }}<x_{\text {máx }}<+\infty\end{array}$ \\
\hline $\begin{array}{l}\text { Beta } \\
\text { (Adaptada) }\end{array}$ & $\begin{aligned} f(x)=\frac{\gamma}{\delta_{1} * \delta_{2}} * \frac{1}{\left(x_{\text {máx }}-x_{\text {mín }}\right)^{\alpha+\beta-1}} *\left(x-x_{\text {min }}\right)^{\alpha-1} \\
*\left(x_{\text {máx }}-x\right)^{\beta-1}\end{aligned}$ & $\begin{array}{c}x_{\min }<x<x_{\operatorname{má} x} \\
\alpha, \beta>0 \\
-\infty<x_{\min } \\
<+\infty\end{array}$ \\
\hline Exponencial & $f(x)=a * e^{-b x}$ & $x \geq 0$ \\
\hline Weibull 3P & $f(x)=\left(\frac{c}{b}\right) *\left(\frac{x-a}{b}\right)^{(c-1)} * e^{\left[-\left(\frac{x-a}{b}\right)^{c}\right]}$ & $\begin{array}{c}x \geq x_{\min } \\
-\infty<x_{\min } \\
<+\infty \\
\mathrm{b}, \mathrm{c}>0 \\
\mathrm{a} \geq 0\end{array}$ \\
\hline Sb de Johnson & $f(x)=\frac{\delta}{\sqrt{2 \pi}} * \frac{\lambda}{(x-\varepsilon)(\lambda+\varepsilon-x)} * e^{\left\{-\frac{1}{2}\left[\gamma+\delta * \ln \left(\frac{x-\varepsilon}{\lambda+\varepsilon-x}\right)\right]^{2}\right\}}$ & $\begin{array}{c}\varepsilon<x<+\lambda \\
-\infty<\gamma<+\infty \\
\lambda>0 \\
\delta>0\end{array}$ \\
\hline Weber & $f(x)=\frac{x^{a}}{(b+c * x)^{d}}$ & $\begin{array}{c}0 \leq x<+\infty \\
d \geq a+1 \\
d \neq a+2 \\
d \neq a+3 \\
a, b, c, d>0\end{array}$ \\
\hline Péllico Netto & $f(x)=\frac{a * x^{2}}{\left(b+c * x^{6}\right)}$ & $\begin{array}{c}x \geq 0 \\
a, b, c>0\end{array}$ \\
\hline
\end{tabular}

$f(x)$ : função de densidade probabilística da variável $x ; x$ : variável aleatória; $\mu$ : média dos valores de $x ; \sigma$ : desvio padrão de $x ; x_{m i n}$ : valor mínimo de $x ; x_{\text {máx }}$ : valor máximo de $x ; \pi: 3,14159 \ldots ; e$ : constante de Euler $(2,718281829 \ldots) ; a, b, c, d, \alpha, \beta, \gamma, \delta, \varepsilon, \lambda$ : parâmetros a serem estimados. 
Sendo assim, para realizar o ajuste das funções, isto é, para a obtenção dos parâmetros das funções de densidade probabilística, foi criada uma planilha no software MS Excel 2007, para obtenção das frequências por classe diamétrica, adotando-se dois intervalos de classes, os quais foram determinados com a amplitude diamétrica, sendo um intervalo de classe de $5 \mathrm{~cm}$ para todas as espécies, bem como para as espécies Ocotea porosa, Araucaria angustifolia, Ocotea elegans e Mimosa scabrella, e um intervalo de classe de $3 \mathrm{~cm}$ para as espécies Myrcia sp., Myrsine gardneriana e Styrax leprosus. Posteriormente, as FDP foram ajustadas com o auxílio da ferramenta "Solver" do MS Excel 2007, o qual utilizou o algoritmo linear de gradiente reduzido generalizado (GRG) na interação dos parâmetros.

Os critérios utilizados para a escolha da FDP de melhor aderência foram as estatísticas de ajuste e precisão, $R_{a j .}^{2}$, $(s y x)$ e (syx \%), calculadas com os valores observados e estimados pelas FDP do número de árvores por classe diamétrica. Além disso, as funções foram submetidas ao teste de Kolmogorov-Smirnov, o qual compara a frequência acumulativa estimada com a frequência acumulativa observada em seu ponto de maior divergência.

Para a seleção das melhores FDP, foi realizada uma classificação com base nas estatísticas de ajuste e precisão e no teste de aderência de Kolmogorov-Smirnov, uma vez que cada função foi classificada de acordo com um ranking composto por todos os critérios citados acima, com a função que apresentou a menor soma sendo selecionada como a FDP de melhor aderência.

\section{RESULTADOS E DISCUSSÃO}

Na tabela 2 estão dispostas as estatísticas descritivas do diâmetro à altura do peito para todas as espécies, bem como para as sete espécies com maior valor de importância em um fragmento de Floresta Ombrófila Mista, as quais foram selecionadas para o ajuste das funções de densidade probabilísticas.

Tabela 2. Estatísticas descritivas do diâmetro à altura do peito $(d)$ para todas as espécies e para as sete espécies com maior valor de importância em um fragmento de Floresta Ombrófila Mista.

Table 2. Descriptive statistics of the diameter at breast height $(d)$ for all species and seven species with the highest importance value in a fragment of Mixed Ombrophyllous Forest.

\begin{tabular}{ccccccccc}
\hline Estatísticas & $\begin{array}{c}\text { Todas as } \\
\text { espécies }\end{array}$ & Myrcia sp. & $\begin{array}{c}\boldsymbol{O} . \\
\text { porosa }\end{array}$ & $\begin{array}{c}\boldsymbol{A} . \\
\text { angustifolia }\end{array}$ & $\begin{array}{c}\boldsymbol{O} . \\
\text { elegans }\end{array}$ & $\begin{array}{c}\text { M. } \\
\text { gardneriana }\end{array}$ & $\begin{array}{c}\text { M. } \\
\text { scabrella }\end{array}$ & $\begin{array}{c}\boldsymbol{S} . \\
\text { leprosus }\end{array}$ \\
\hline $\bar{d}$ & 13,1 & 9,1 & 20,3 & 19,6 & 15,7 & 10,2 & 15,9 & 12,1 \\
$\sigma^{2}$ & 91,1 & 15,6 & 248,0 & 188,9 & 97,6 & 26,0 & 133,7 & 27,2 \\
$\sigma$ & 9,5 & 3,9 & 15,7 & 13,7 & 9,9 & 5,1 & 11,6 & 5,2 \\
$d_{\text {máx. }}$ & 100,3 & 29,9 & 100,3 & 56,0 & 55,1 & 27,9 & 52,5 & 27,2 \\
$d_{\text {mín. }}$ & 5,0 & 5,1 & 5,1 & 5,1 & 5,3 & 5,1 & 5,3 & 5,1 \\
$A$ & 95,2 & 24,8 & 95,2 & 50,9 & 49,8 & 22,8 & 47,3 & 22,1 \\
$I_{c}$ & 5 & 3 & 5 & 5 & 5 & 3 & 5 & 3 \\
\hline $\bar{d}$
\end{tabular}

$\bar{d}$ : diâmetro médio $(\mathrm{cm}) ; \sigma^{2}$ : variância $\left(\mathrm{cm}^{2}\right) ; \sigma$ : desvio padrão $(\mathrm{cm}) ; d_{\text {máx }}$ : diâmetro máximo $(\mathrm{cm}) ; d_{\text {mín }}$ : diâmetro mínimo $(\mathrm{cm})$; $A$ : amplitude diamétrica $(\mathrm{cm}) ; I_{c}$ : intervalo de classe $(\mathrm{cm})$.

Na tabela 3 está representado o número de árvores por hectare, por classe diamétrica, em relação à frequência observada, com intervalo de classe de $5 \mathrm{~cm}$, considerando todas as espécies do fragmento florestal, bem como para as espécies Ocotea porosa, Araucaria angustifolia, Ocotea elegans e Mimosa scabrella.

Para todas as espécies, bem como por espécie, observa-se que o maior número de árvores do fragmento está concentrado nas duas primeiras classes de diâmetro, cuja variação é de 5 a $15 \mathrm{~cm}$. Verifica-se que, com o aumento das classes diamétricas, geralmente o número de árvores diminui expressivamente. Por outro lado, é possível observar um aumento da frequência da espécie Araucaria angustifolia na classe diamétrica com valor central de $37,5 \mathrm{~cm}$, para Ocotea porosa na classe diamétrica de valor central de $22,5 \mathrm{~cm}$ e para Ocotea elegans na classe diamétrica com valor de $32,5 \mathrm{~cm}$.

$\mathrm{Na}$ tabela 4 consta o número de árvores por hectare, por classe diamétrica, em relação à frequência observada, com intervalo de classe de $3 \mathrm{~cm}$ para as espécies Myrcia sp., Myrsine gardneriana e Styrax leprosus.

Observa-se que, para os indivíduos de Myrcia sp., Myrsine gardneriana e Styrax leprosus, o maior número de árvores está concentrado nas duas primeiras classes de diâmetro, que varia de 5 a $11 \mathrm{~cm}$. 
Tabela 3. Número de árvores por hectare, por classe diamétrica, considerando todas as espécies do fragmento florestal e para as espécies com maior valor de importância, com intervalo de classe de $5 \mathrm{~cm}$, em um fragmento de Floresta Ombrófila Mista.

Table 3. Number of trees per hectare by diameter class, considering all species of the forest and species with the highest importance value, with class width of $5 \mathrm{~cm}$, in a fragment of Mixed Ombrophyllous Forest.

\begin{tabular}{lccccc}
\hline Centro de classe & $\begin{array}{c}\text { Todas as } \\
\text { espécies }\end{array}$ & O. porosa & A. angustifolia & O. elegans & M. scabrella \\
\hline 7,5 & 876 & 46 & 48 & 37 & 20 \\
12,5 & 385 & 33 & 16 & 21 & 19 \\
17,5 & 169 & 17 & 10 & 14 & 6 \\
22,5 & 103 & 22 & 8 & 14 & 3 \\
27,5 & 61 & 13 & 9 & 4 & 3 \\
32,5 & 37 & 9 & 6 & 6 & - \\
37,5 & 35 & 7 & 12 & 5 & 1 \\
42,5 & 13 & 3 & 7 & - & 2 \\
47,5 & 8 & 2 & 3 & - & 1 \\
52,5 & 7 & 3 & 1 & - & - \\
57,5 & 3 & 1 & 1 & 1 & - \\
62,5 & 3 & 2 & - & - & - \\
67,5 & 1 & 1 & - & - & - \\
72,5 & - & - & - & - & - \\
77,5 & - & - & - & - & - \\
82,5 & - & - & - & - & - \\
87,5 & - & - & - & - & - \\
92,5 & 1 & 1 & - & - & - \\
97,5 & - & - & - & - & - \\
102,5 & 1 & 1 & 122 & 102 & \\
\hline
\end{tabular}

De acordo com Schaaf et al. (2006), Machado et al. (2009a), Figueiredo Filho et al. (2010) e Machado et al. (2010a), estudando fragmentos de Floresta Ombrófila Mista em diferentes regiões no estado do Paraná, foi encontrada maior concentração de indivíduos nas duas primeiras classes diamétricas e diminuição do número de indivíduos nas classes subsequentes, o que proporcionou classes diamétricas intermediárias sem árvores. Indicaram uma distribuição diamétrica decrescente, resultado muito semelhante ao encontrado no presente estudo.

Tabela 4. Número de árvores por hectare, por classe diamétrica, para as espécies com maior valor de importância, com intervalo de classe de $3 \mathrm{~cm}$, em um fragmento de Floresta Ombrófila Mista.

Table 4. Number of trees per hectare by diameter class for the species with the highest importance value, with class interval of $3 \mathrm{~cm}$, in a fragment of Mixed Ombrophyllous Forest.

\begin{tabular}{lccc}
\hline Centro de Classe & Myrcia sp. & M. gardneriana & S. leprosus \\
\hline 6,5 & 242 & 54 & 23 \\
9,5 & 127 & 26 & 17 \\
12,5 & 58 & 14 & 18 \\
15,5 & 20 & 6 & 10 \\
18,5 & 14 & 9 & 4 \\
21,5 & 4 & 2 & 2 \\
24,5 & 2 & 1 & 1 \\
27,5 & 4 & 3 & - \\
30,5 & 1 & - & 85 \\
\hline
\end{tabular}

Ajuste das funções de densidade probabilísticas

Para o ajuste das FDP, são apresentados os parâmetros estimados, os quais são utilizados diretamente nessas funções para obtenção da frequência de árvores por classe diamétrica para todas as

FLORESTA, Curitiba, PR, v. 45, n. 2, p. 337 - 348, abr. / jun. 2015.

Téo, S. J. et al.

ISSN eletrônico 1982-4688 / ISSN impresso 0015-3826

DOI: $10.5380 /$ rf.v45i2.34733 
espécies, bem como para as sete espécies estudadas (Tabela 5).

Tabela 5. Parâmetros estimados das FDP para todas as espécies e para as sete espécies com maior valor de importância em um fragmento de Floresta Ombrófila Mista.

Table 5. Estimated parameters of the PDF for all species and seven species with the highest importance value, in a fragment of Mixed Ombrophyllous Forest.

\begin{tabular}{|c|c|c|c|c|c|c|c|c|c|}
\hline Função & Parâmetros & $\begin{array}{l}\text { Todas as } \\
\text { espécies }\end{array}$ & Myrcia sp. & $\begin{array}{c}O . \\
\text { porosa }\end{array}$ & $\begin{array}{c}A . \\
\text { angustifolia }\end{array}$ & $\begin{array}{c}O . \\
\text { elegans }\end{array}$ & $\begin{array}{c}M . \\
\text { gardneriana }\end{array}$ & $\begin{array}{c}M . \\
\text { scabrella } \\
\end{array}$ & $\begin{array}{c}S . \\
\text { leprosus } \\
\end{array}$ \\
\hline \multirow{3}{*}{ Normal } & $\mu$ & $-128,051$ & $-8,70781$ & $-157,78$ & $-151,46401$ & $-149,775$ & $-60,66496$ & 9,54253 & 3,77037 \\
\hline & $\sigma^{2}$ & 895,1654 & 76,61731 & 2717,45 & 1198,76860 & 1971,778 & 325,17255 & 28,25616 & 100,9746 \\
\hline & $\sigma$ & 0,00014 & 0,51560 & 0,04720 & 0,00014 & 0,01081 & 0,00252 & 5,24477 & 4,44138 \\
\hline \multirow{3}{*}{ Ln-normal } & $\mu$ & 1,70501 & 1,88303 & 2,59950 & $-29,46085$ & 2,32910 & 0,90237 & 2,40860 & 2,37486 \\
\hline & $\sigma^{2}$ & 0,87380 & 0,25662 & 0,94253 & 64,02670 & 1,01071 & 1,21168 & 0,16523 & 0,42424 \\
\hline & $\sigma$ & 0,48900 & 0,35931 & 0,78224 & 0,00031 & 0,70583 & 0,26619 & 0,47162 & 0,52529 \\
\hline \multirow{3}{*}{$\begin{array}{l}\text { Gamma } \\
\text { (Adaptada) }\end{array}$} & $\alpha$ & 0,68720 & 1,25682 & 0,93573 & 0,15496 & 0,65563 & 0,70134 & 5,83600 & 1,26502 \\
\hline & $\beta$ & 9,81400 & 3,19662 & 16,7848 & 3878,8719 & 16,00816 & 7,23624 & 1,95814 & 7,09856 \\
\hline & $\gamma$ & 1,18378 & 0,95525 & 1,02424 & 1,70792 & 0,70784 & 1,18584 & 103,843 & 0,85796 \\
\hline \multirow{5}{*}{$\begin{array}{l}\text { Beta } \\
\text { (Adaptada) }\end{array}$} & $\alpha$ & 0,60056 & 1,01031 & 0,84193 & 0,14866 & 0,69185 & 0,51352 & 2,06408 & 1,00404 \\
\hline & $\beta$ & 8,60321 & 5,84438 & 4,89114 & 0,94939 & 2,63939 & 2,48653 & 12,24036 & 2,15553 \\
\hline & $\gamma$ & 1,11928 & 1,85668 & 1,73094 & 0,37895 & 0,90516 & 2,94435 & 4,26778 & 1,15717 \\
\hline & $\delta_{1}$ & 0,63368 & 0,56601 & 0,71684 & 1,13835 & 0,77794 & 1,70554 & 0,17026 & 0,73615 \\
\hline & $\delta_{2}$ & 0,63368 & 0,56601 & 0,71684 & 1,13835 & 0,77794 & 1,70554 & 0,17026 & 0,73615 \\
\hline \multirow{2}{*}{ Exponencial } & $\mathrm{a}$ & 9,52741 & 6,94422 & 4,16190 & 4,57467 & 4,13322 & 5,22199 & 3,65932 & 3,73609 \\
\hline & $\mathrm{b}$ & $-0,40169$ & $-0,23838$ & $-0,0660$ & $-0,12501$ & $-0,08556$ & $-0,21843$ & $-0,09765$ & $-0,09536$ \\
\hline \multirow{3}{*}{ Weibull 3P } & $\mathrm{a}$ & 5,37163 & 4,88654 & 5,30581 & 5,57288 & 5,68098 & 5,31545 & $-2,13536$ & 5,18448 \\
\hline & $\mathrm{b}$ & 6,74130 & 4,14615 & 15,5895 & 13,03807 & 12,57431 & 5,03976 & 13,73533 & 8,69929 \\
\hline & $\mathrm{c}$ & 0,85053 & 1,12929 & 0,96540 & 0,89489 & 0,92320 & 0,84796 & 2,58289 & 1,19180 \\
\hline \multirow{4}{*}{$\begin{array}{l}\mathrm{Sb} \text { de } \\
\text { Johnson }\end{array}$} & $\varepsilon$ & 4,32963 & 3,92968 & 3,97587 & 5,63109 & 4,58401 & 4,90950 & $-12,88726$ & 4,25884 \\
\hline & $\lambda$ & 872,2933 & 65,11435 & 91,9542 & 48,98632 & 52,86819 & 93,18498 & 730,5606 & 21,81337 \\
\hline & $\delta$ & 0,96094 & 1,26597 & 0,81109 & 0,47447 & 0,74883 & 0,82548 & 4,21685 & 0,77181 \\
\hline & $\gamma$ & 4,82041 & 3,44003 & 1,60327 & 0,64353 & 1,21839 & 2,57413 & 14,41573 & 0,62190 \\
\hline \multirow{4}{*}{ Weber } & $\mathrm{a}$ & 1,84759 & 2,40817 & $-0,1334$ & $-2,13907$ & $-0,34311$ & $-1,44994$ & 5,26367 & 1,35646 \\
\hline & $\mathrm{b}$ & 0,96357 & 1,06274 & 1,00527 & 0,97025 & 0,99864 & 0,97732 & 1,08756 & 1,02337 \\
\hline & $\mathrm{c}$ & 0,16197 & 0,03020 & 0,00057 & $-0,00037$ & 0,00061 & 0,00063 & 0,00739 & 0,00201 \\
\hline & $\mathrm{d}$ & 5,63444 & 22,47101 & 103,976 & 101,72020 & 103,0146 & 104,42971 & 87,16841 & 108,5803 \\
\hline \multirow{3}{*}{ Pélico Netto } & $\mathrm{a}$ & 1,90085 & 147,02342 & 0,06404 & 0,41150 & 31,26925 & 118,17847 & 27,56587 & 34,30631 \\
\hline & $\mathrm{b}$ & 793,0329 & 24085,8710 & 58,7605 & 210,04914 & 21209,10 & 20999,8309 & 20000,01 & 21209,02 \\
\hline & $\mathrm{c}$ & 0,00140 & 0,16336 & 0,00004 & 0,00048 & 0,01994 & 0,14693 & 0,01175 & 0,01585 \\
\hline
\end{tabular}

Convém ressaltar que as funções de densidade probabilísticas foram ajustadas com o auxílio da ferramenta "Solver" do MS Excel 2007, que utiliza o algoritmo GRG. Sendo assim, observou-se que os parâmetros de algumas FDP apresentaram valores não usualmente encontrados utilizando-se outros métodos de ajuste, como, por exemplo, o método dos momentos. Tais valores não usuais foram fornecidos pelo referido algoritmo matemático por resultar na menor soma de quadrados dos resíduos. Um exemplo desse comportamento é a distribuição Normal, cujos valores para o parâmetro " $\mu$ " foram negativos, os quais, obviamente, não representam a média real dos diâmetros das árvores amostradas para a classificação diamétrica neste trabalho.

Na tabela 6 é possível observar o desempenho das FDP para todas as espécies e para as sete espécies com maior valor de importância em um fragmento de Floresta Ombrófila Mista.

Analisando as estatísticas de ajuste e precisão, bem como os valores de $d_{\text {calc. }}$ do teste de Kolmogorov-Smirnov, observa-se que a função Sb de Johnson apresentou melhor desempenho para descrever a distribuição diamétrica quando consideradas todas as espécies do fragmento de Floresta Ombrófila Mista. Quando consideradas individualmente as espécies Myrcia sp., Ocotea porosa, Araucaria angustifolia, Ocotea elegans, Myrsine gardneriana, Mimosa scabrella e Styrax leprosus, as funções de melhor desempenho foram Ln-normal, Gamma (Adaptada), Gamma (Adaptada), Weibull 3P, Ln-normal, Péllico Netto e Normal, respectivamente. 
Tabela 6. Desempenho das FDP para todas as espécies e para as sete espécies com maior valor de importância em um fragmento de Floresta Ombrófila Mista.

Table 6. Performance of PDF for all species and seven species with the highest importance value, in a fragment of Mixed Ombrophyllous Forest.

\begin{tabular}{|c|c|c|c|c|c|c|c|}
\hline FDP & Espécie & Ranking & $R_{a j}^{2}$ & syx (árv/ha) & syx $\%$ & $d_{t a b}$ & $d_{\text {calc. }}$ \\
\hline Sb de Johnson & & $1^{\circ}$ & $\begin{array}{c}\text { aj. } \\
0,9994\end{array}$ & 5,08 & 5,96 & & $0,00733^{\text {ns }}$ \\
\hline Weber & & $2^{\circ}$ & 0,9993 & 5,40 & 6,34 & & $0,00799^{\text {ns }}$ \\
\hline Ln-normal & & $3^{\circ}$ & 0,9993 & 5,45 & 6,40 & & $0,00735^{\text {ns }}$ \\
\hline Weibull 3P & & $4^{\circ}$ & 0,9989 & 7,00 & 8,22 & & $0,01186^{\mathrm{ns}}$ \\
\hline Gamma (Adaptada) & Todas as espécies & $5^{\circ}$ & 0,9987 & 7,53 & 8,84 & 0,03950 & $0,01505^{\text {ns }}$ \\
\hline Beta (Adaptada) & & $6^{\circ}$ & 0,9980 & 9,32 & 10,94 & & $0,02233^{\text {ns }}$ \\
\hline Normal & & $7^{\circ}$ & 0,9953 & 14,20 & 16,67 & & $0,06741 * *$ \\
\hline Pélico Netto & & $8^{\circ}$ & 0,9865 & 24,05 & 28,24 & & $0,11877 * *$ \\
\hline Exponencial & & $9^{\circ}$ & 0,9842 & 26,07 & 30,61 & & $0,35142 * *$ \\
\hline Ln-normal & & $1^{\circ}$ & 0,9990 & 2,64 & 5,04 & & $0,00665^{\mathrm{ns}}$ \\
\hline Gamma (Adaptada) & & $2^{\circ}$ & 0,9989 & 2,77 & 5,29 & & $0,01050^{\mathrm{ns}}$ \\
\hline Sb de Johnson & & $3^{\circ}$ & 0,9986 & 3,03 & 5,77 & & $0,005911^{\mathrm{ns}}$ \\
\hline Weber & & $4^{\circ}$ & 0,9987 & 2,98 & 5,69 & & $0,00901^{\mathrm{ns}}$ \\
\hline Weibull 3P & Myrcia sp. & $5^{\circ}$ & 0,9988 & 2,86 & 5,45 & 0,07503 & $0,01240^{\text {ns }}$ \\
\hline Normal & & $6^{\circ}$ & 0,9985 & 3,22 & 6,14 & $0,0 / 500$ & $0,01815^{\mathrm{ns}}$ \\
\hline Pélico Netto & & $7^{\circ}$ & 0,9977 & 3,97 & 7,56 & & $0,01231^{\text {ns }}$ \\
\hline Beta (Adaptada) & & $8^{\circ}$ & 0,9970 & 4,47 & 8,52 & & $0,024911^{\text {ns }}$ \\
\hline Exponencial & & $9^{\circ}$ & 0,9968 & 4,65 & 8,86 & & $0,02026^{\text {ns }}$ \\
\hline Gamma (Adaptada) & & $1^{\circ}$ & 0,9720 & 2,10 & 26,15 & & $0,02798^{\mathrm{ns}}$ \\
\hline Weibull 3P & & $2^{\circ}$ & 0,9719 & 2,11 & 26,22 & & $0,01523^{\mathrm{ns}}$ \\
\hline Exponencial & & $3^{\circ}$ & 0,9728 & 2,08 & 25,79 & & $0,03323^{\mathrm{ns}}$ \\
\hline Normal & & $4^{\circ}$ & 0,9702 & 2,17 & 27,00 & & $0,03307^{\mathrm{ns}}$ \\
\hline Weber & O. porosa & $5^{\circ}$ & 0,9700 & 2,18 & 27,08 & 0,12846 & $0,02879^{\mathrm{ns}}$ \\
\hline Beta (Adaptada) & 0. porosa & $6^{\circ}$ & 0,9696 & $\begin{array}{l}2,10 \\
2,19\end{array}$ & $\begin{array}{l}27,00 \\
27,24\end{array}$ & 0,12040 & $0,02529^{\mathrm{ns}}$ \\
\hline Ln-normal & & $7^{\circ}$ & 0,9681 & 2,25 & 27,92 & & $0,03187^{\mathrm{ns}}$ \\
\hline Sb de Johnson & & $8^{\circ}$ & 0,9680 & 2,25 & 27,99 & & $0,03138^{\mathrm{ns}}$ \\
\hline Pélico Netto & & $9^{\circ}$ & 0,7861 & 5,82 & 72,32 & & $0,32873 * *$ \\
\hline Gamma (Adaptada) & & $1^{\circ}$ & 0,9414 & 3,15 & 28,31 & & $0,05787^{\mathrm{ns}}$ \\
\hline Beta (Adaptada) & & $2^{\circ}$ & 0,9254 & 3,56 & 31,95 & & $0,05084^{\mathrm{ns}}$ \\
\hline Weber & & $3^{\circ}$ & 0,9346 & 3,33 & 29,91 & & $0,07617^{\mathrm{ns}}$ \\
\hline Sb de Johnson & & $4^{\circ}$ & 0,9316 & 3,41 & 30,60 & & $0,06329^{\mathrm{ns}}$ \\
\hline Ln-normal & A. angustifolia & $5^{\circ}$ & 0,9029 & 4,06 & 36,46 & 0,14757 & $0,07025^{\mathrm{ns}}$ \\
\hline Weibull 3P & & $6^{\circ}$ & 0,8091 & 5,69 & 51,12 & & $0,12032^{\mathrm{ns}}$ \\
\hline Exponencial & & $7^{\circ}$ & 0,7983 & 5,85 & 52,54 & & $0,24913 * *$ \\
\hline Normal & & $8^{\circ}$ & 0,7605 & 6,37 & 57,24 & & $0,26375 * *$ \\
\hline Pélico Netto & & $9^{\circ}$ & 0,7277 & 6,79 & 61,04 & & $0,37553 * *$ \\
\hline Weibull 3P & & $1^{\circ}$ & 0,9619 & 2,25 & 24,32 & & $0,02630^{\mathrm{ns}}$ \\
\hline Beta (Adaptada) & & $2^{\circ}$ & 0,9583 & 2,36 & 25,44 & & $0,02208^{\mathrm{ns}}$ \\
\hline Gamma (Adaptada) & & $3^{\circ}$ & 0,9612 & 2,28 & 24,55 & & $0,02910^{\mathrm{ns}}$ \\
\hline Exponencial & & $4^{\circ}$ & 0,9609 & 2,22 & 23,99 & & $0,03158^{\mathrm{ns}}$ \\
\hline Sb de Johnson & O. elegans & $5^{\circ}$ & 0,9572 & 2,39 & 25,76 & 0,16139 & $0,02272^{\mathrm{ns}}$ \\
\hline Normal & & $6^{\circ}$ & 0,9562 & 2,42 & 26,08 & & $0,02790^{\mathrm{ns}}$ \\
\hline Ln-normal & & $7^{\circ}$ & 0,9564 & 2,41 & 26,00 & & $0,03506^{\mathrm{ns}}$ \\
\hline Weber & & $8^{\circ}$ & 0,9555 & 2,44 & 26,28 & & $0,02929^{\mathrm{ns}}$ \\
\hline Pélico Netto & & $9^{\circ}$ & 0,7836 & 5,37 & 57,95 & & $0,25229 * *$ \\
\hline Ln-normal & & $1^{\circ}$ & 0,9844 & 2,25 & 15,63 & & $0,01738^{\text {ns }}$ \\
\hline Weibull 3P & & $2^{\circ}$ & 0,9837 & 2,30 & 15,97 & & $0,01968^{\mathrm{ns}}$ \\
\hline Sb de Johnson & & $3^{\circ}$ & 0,9807 & 2,50 & 17,41 & & $0,01668^{\mathrm{ns}}$ \\
\hline Gamma (Adaptada) & & $4^{\circ}$ & 0,9834 & 2,31 & 16,10 & & $0,02075^{\mathrm{ns}}$ \\
\hline Weber & M. gardneriana & $5^{\circ}$ & 0,9803 & 2,53 & 17,57 & 0,15200 & $0,01755^{\text {ns }}$ \\
\hline Exponencial & & $6^{\circ}$ & 0,9805 & 2,51 & 17,48 & & $0,06264^{\mathrm{ns}}$ \\
\hline Normal & & $7^{\circ}$ & 0,9727 & 2,97 & 20,69 & & $0,04831^{\mathrm{ns}}$ \\
\hline Beta (Adaptada) & & $8^{\circ}$ & 0,9645 & 3,39 & 23,58 & & $0,02807^{\mathrm{ns}}$ \\
\hline Pélico Netto & & $9^{\circ}$ & 0,9578 & 3,70 & 25,71 & & $0,10506^{\mathrm{ns}}$ \\
\hline Pélico Netto & & $1^{\circ}$ & 0,9687 & 1,32 & 23,13 & & $0,10911^{\mathrm{ns}}$ \\
\hline Ln-normal & & $2^{\circ}$ & 0,9553 & 1,58 & 27,65 & & $0,13236^{\mathrm{ns}}$ \\
\hline Gamma (Adaptada) & & $3^{\circ}$ & $\begin{array}{l}0,933 \\
0,9488\end{array}$ & $\begin{array}{l}1,00 \\
1,69\end{array}$ & $\begin{array}{l}27,03 \\
29,60\end{array}$ & & $0,14538^{\mathrm{ns}}$ \\
\hline Normal & & $4^{\circ}$ & $\begin{array}{l}0,930 \\
0,9396\end{array}$ & $\begin{array}{l}1,09 \\
1,83\end{array}$ & 32,14 & & $0,01826^{\mathrm{ns}}$ \\
\hline Weber & M. scabrella & $5^{\circ}$ & 0,9416 & 1,80 & 31,60 & 0,21590 & $0,14699^{\mathrm{ns}}$ \\
\hline Sb de Johnson & & $6^{\circ}$ & 0,9349 & $\begin{array}{l}1,00 \\
1,90\end{array}$ & 33,37 & & $0,14612^{\text {ns }}$ \\
\hline Exponencial & & $7^{\circ}$ & 0,8848 & 2,53 & 44,38 & & $0,05907^{\mathrm{ns}}$ \\
\hline Weibull 3P & & $8^{\circ}$ & 0,9382 & 1,85 & 32,51 & & $0,15884^{\mathrm{ns}}$ \\
\hline Beta (Adaptada) & & $9^{\circ}$ & 0,9270 & 2,01 & 35,33 & & $0,15605^{\mathrm{ns}}$ \\
\hline Normal & & $1^{\circ}$ & 0,9365 & 2,04 & 19,23 & & $0,01542^{\mathrm{ns}}$ \\
\hline Sb de Johnson & & $2^{\circ}$ & 0,9163 & 2,34 & 22,07 & & $0,01865^{\mathrm{ns}}$ \\
\hline Weibull 3P & & $3^{\circ}$ & 0,9120 & 2,40 & 22,63 & & $0,04133^{\mathrm{ns}}$ \\
\hline Gamma (Adaptada) & & $4^{\circ}$ & 0,9071 & 2,47 & 23,26 & & $0,03145^{\mathrm{ns}}$ \\
\hline Beta (Adaptada) & S. leprosus & $5^{\circ}$ & 0,9022 & 2,54 & 23,86 & 0,17680 & $0,01812^{\mathrm{ns}}$ \\
\hline Ln-normal & & $6^{\circ}$ & 0,9040 & 2,51 & $\begin{array}{l}25,00 \\
23,64\end{array}$ & & $0,03169^{\mathrm{ns}}$ \\
\hline Weber & & $7^{\circ}$ & 0,9005 & 2,56 & 24,07 & & $0,02468^{\mathrm{ns}}$ \\
\hline Exponencial & & $8^{\circ}$ & 0,8963 & 2,61 & 24,56 & & $0,07101^{\mathrm{ns}}$ \\
\hline Pélico Netto & & $9^{\circ}$ & 0,6134 & 5,04 & 47,43 & & $0,11084^{\mathrm{ns}}$ \\
\hline
\end{tabular}

FDP: função de densidade probabilística; $R_{a j}^{2}$ : coeficiente de determinação ajustado; syx: erro padrão de estimativa, em arv./ha; syx\%: erro padrão de estimativa em porcentagem; $d_{t a b .}$ : valor tabelado do teste de Kolmogorov-Smirnov $(\alpha=0,01) ; d_{c a l c}$ : valor calculado do teste de Kolmogorov-Smirnov; ${ }^{\text {ns: }}$ não significativo ao nível de $1 \%(\alpha=0,01)$; **: significativo ao nível de $1 \%(\alpha=0,01)$.

FLORESTA, Curitiba, PR, v. 45, n. 2, p. 337 - 348, abr. / jun. 2015.

Téo, S. J. et al.

ISSN eletrônico 1982-4688 / ISSN impresso 0015-3826 
Convém salientar que, por meio dos critérios citados acima, observa-se que a função Exponencial apresentou o pior desempenho para descrever a distribuição diamétrica quando consideradas todas as espécies do fragmento de Floresta Ombrófila Mista. No entanto, quando consideradas individualmente as espécies Myrcia sp., Ocotea porosa, Araucaria angustifolia, Ocotea elegans, Myrsine gardneriana, Mimosa scabrella e Styrax leprosus, as funções de pior desempenho foram Exponencial, Péllico Netto, Péllico Netto, Péllico Netto, Péllico Netto, Beta (Adaptada) e Péllico Netto, respectivamente.

Por meio dos resultados das estatísticas de ajuste e precisão, as funções com melhor desempenho apresentaram altos valores de $R_{a j}^{2}$, e baixos valores de $s y x$ e $s y x \%$, sendo semelhantes aos estudos encontrados por Machado et al. (2009a) e Machado et al. (2010a) em um fragmento de Floresta Ombrófila Mista em Curitiba, PR. Por outro lado, algumas FDP, em alguns casos, apresentaram valores muito altos para $s y x \%$, os quais são devido à baixa frequência média nas classes diamétricas.

Em relação aos valores de $d_{\text {calc. }}$ do teste de Kolmogorov-Smirnov, os ajustes que apresentaram valores não significativos indicam a aceitação da hipótese de nulidade, o que significa que as frequências esperadas e observadas são similares do ponto de vista estatístico, descrevendo bem o conjunto de dados. No entanto, as FDP que apresentaram valores significativos não apresentaram boa aderência aos dados de frequência observados.

De maneira geral, quando considerada a distribuição diamétrica para todas as espécies do fragmento florestal estudado e cada espécie separadamente, a FDP de melhor desempenho foi a função Gamma (Adaptada), seguida da função Ln-Normal, Weibull 3P e Sb de Johnson, para as quais o teste de Kolmogorov-Smirnov sempre indicou boa aderência aos resultados da distribuição diamétrica observada. Por outro lado, as funções Exponencial e de Péllico Netto, também considerando-se todas as espécies do fragmento florestal e cada espécie separadamente, apresentaram os piores desempenhos, para as quais o teste de Kolmogorov-Smirnov não indicou boa aderência aos resultados da distribuição diamétrica observada, em alguns casos, conforme apresentado na Tabela 5.

Bartoszeck et al. (2004), Orellana (2009), Machado et al. (2009a), Machado et al. (2009b), Machado et al. (2010a) e Machado et al. (2010b), estudando fragmentos de Floresta Ombrófila Mista em diferentes regiões do Estado do Paraná, por meio dos resultados do teste de Kolmogorov-Smirnov, indicaram as funções Sb de Johnson, Gamma (Adaptada), Weibull 3P, Normal e Weber como as que proporcionaram maior eficiência para representar a distribuição diamétrica, resultados semelhantes aos encontrados neste estudo em relação às quatro primeiras funções citadas.

Na figura 1, está ilustrada a curva de distribuição diamétrica estimada por meio da melhor FDP ajustada considerando todas as espécies do fragmento florestal em relação ao histograma de frequência observada.

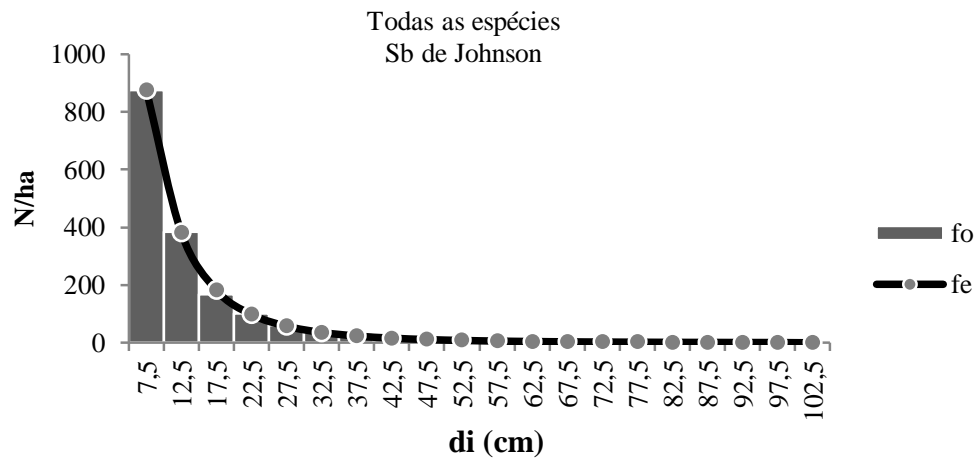

Figura 1. Curva de distribuição diamétrica estimada sobre a distribuição diamétrica observada, considerando todas as espécies do fragmento de Floresta Ombrófila Mista da região de Lebon Régis, SC.

Figure 1. Estimated Diameter distribution curve on the observed diameter distribution, considering all species of the Mixed Ombrophyllous Forest fragment, in the region Lebon Régis, SC.

Considerando todas as espécies do fragmento florestal, ao comparar a distribuição diamétrica da menor para a maior classe diamétrica, verificou-se uma grande concentração de indivíduos nas classes de menores diâmetros, constatando-se a característica do formato de distribuição diamétrica decrescente (ou J-invertido), típico da estrutura diamétrica para florestas mistas, com várias espécies e várias classes de

FLORESTA, Curitiba, PR, v. 45, n. 2, p. 337 - 348, abr. / jun. 2015. 
idade, percebendo-se a existência de poucas árvores com mais de $47,5 \mathrm{~cm}$ de diâmetro à altura do peito.

Na figura 2 estão ilustradas as curvas de distribuição diamétrica estimadas por meio das melhores FDP ajustadas, com intervalo de classe de $5 \mathrm{~cm}$, considerando as espécies Ocotea porosa, Araucaria angustifolia, Ocotea elegans e Mimosa scabrella, e com intervalo de classe de $3 \mathrm{~cm}$, considerando as espécies Myrcia sp., Myrsine gardneriana e Styrax leprosus, em relação ao histograma de frequência observada.
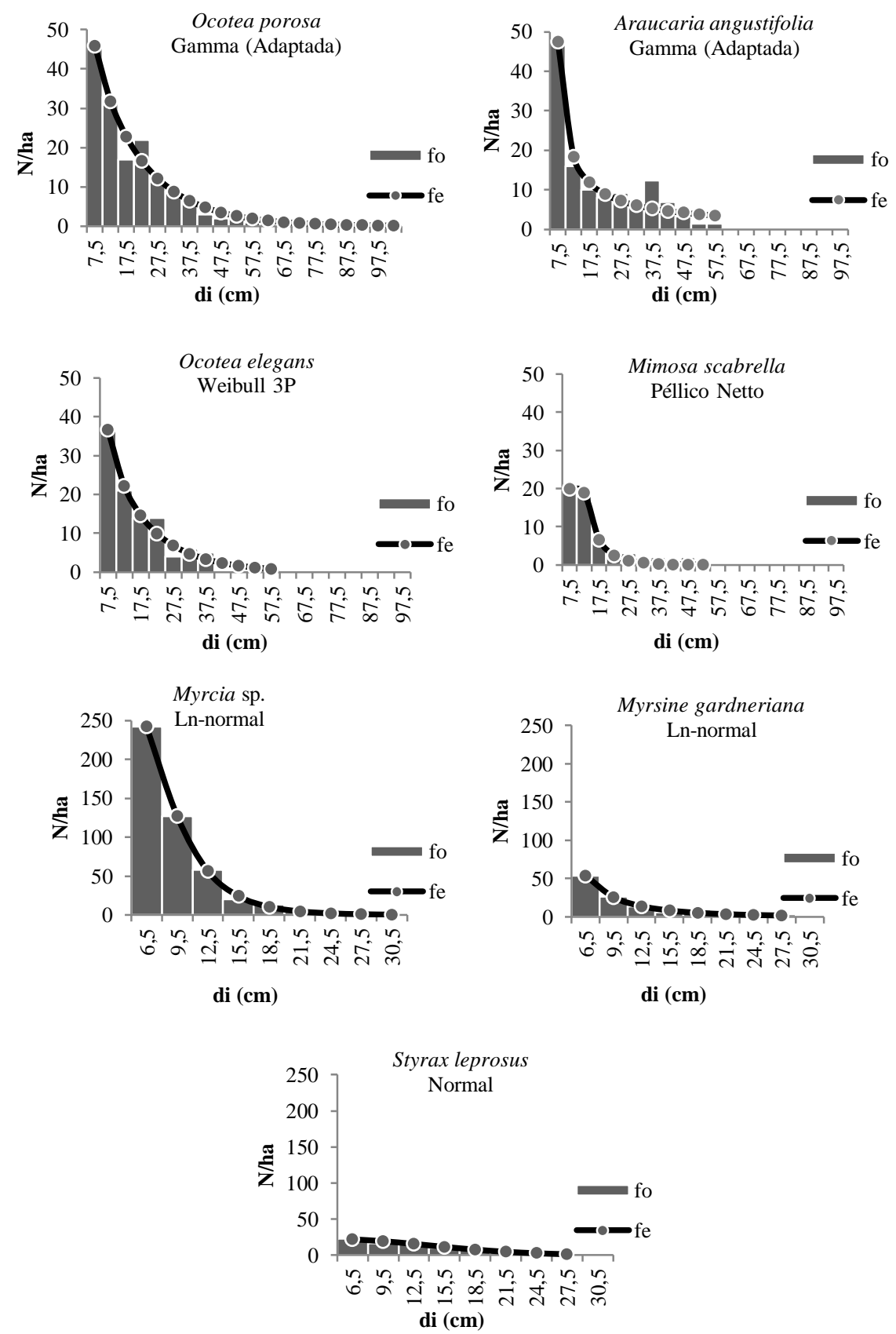

Figura 2. Curvas de distribuição diamétrica estimadas sobre a distribuição diamétrica observada, para as espécies com intervalo de classe de $5 \mathrm{~cm}$ e $3 \mathrm{~cm}$ em fragmento de Floresta Ombrófila Mista na região de Lebon Régis, SC.

Figure 2. Estimated Diameter distribution curves on the observed diameter distribution, for the species with class interval of $5 \mathrm{~cm}$ and $3 \mathrm{~cm}$ of fragment of Mixed Ombrophyllous Forest, the region Lebon Régis, SC.

FLORESTA, Curitiba, PR, v. 45, n. 2, p. 337 - 348, abr. / jun. 2015.

Téo, S. J. et al.

ISSN eletrônico 1982-4688 / ISSN impresso 0015-3826 
Convém ressaltar que todas as espécies apresentaram distribuição diamétrica decrescente, no entanto algumas de forma menos acentuada, como é o caso da espécie Styrax leprosus.

A distribuição diamétrica decrescente também foi constatada por Schaaf et al. (2006), Orellana (2009), Machado et al. (2009a), Figueiredo Filho et al. (2010) e Machado et al. (2010a) estudando fragmentos de Floresta Ombrófila Mista no estado do Paraná.

Meyer et al. (1961), quando introduziram o termo "floresta balanceada", com o propósito de garantir um rendimento sustentável, relataram que a distribuição diamétrica em formações florestais pode ser muitas vezes descontínua, não seguindo realmente a forma J-invertido e muito menos ser balanceada, o que é característico do presente estudo, principalmente quando analisadas as espécies individualmente.

A distribuição diamétrica das espécies Ocotea porosa, Araucaria angustifolia, Ocotea elegans e Mimosa scabrella apresentam-se, em grade parte, com tendência decrescente. No entanto, percebe-se que algumas classes de diâmetro apresentam maiores valores de frequência com relação às classes anteriores. Esse comportamento é mais evidente para a distribuição diamétrica de Araucaria angustifolia (Figura 2).

Machado et al. (1996) e Machado et al. (2009b), utilizando dados de um grande número de parcelas medidas e de censo florestal, respectivamente, para descrever a distribuição diamétrica de Araucaria angustifolia em fragmentos de Floresta Ombrófila Mista, no Sul do Brasil, encontraram distribuições unimodais para Araucaria angustifolia. Machado et al. (1996) utilizaram somente dados de diâmetro acima de $20 \mathrm{~cm}$, provenientes de florestas secundárias de diversas idades e graus de interferência humana. Já Machado et al. (2009b) realizaram censo em um fragmento florestal que sofreu exploração seletiva no passado, o que pode ter afetado a regeneração da Araucaria angustifolia.

\section{CONCLUSÕES}

De acordo com os resultados da presente pesquisa foi possível concluir que:

- A distribuição diamétrica do fragmento de Floresta Ombrófila Mista na região de Lebon Régis, Santa Catarina, foi decrescente, indicando um elevado grau de regeneração natural.

- A função Sb de Johnson apresentou melhor ajuste para a distribuição diamétrica considerando todas as espécies do fragmento de Floresta Ombrófila Mista em Lebon Régis, SC.

- De modo geral, as funções Gamma (Adaptada), Ln-normal, Weibull 3P e Sb de Johnson propiciaram os melhores desempenhos para as espécies estudadas, e a função Exponencial e de Péllico Netto proporcionaram pior desempenho.

\section{REFERÊNCIAS}

ARCE, J. E. Modelagem da estrutura de florestas clonais de Populus deltoides March através de distribuições diamétricas probabilísticas. Ciência Florestal, Santa Maria, v. 14, n. 1, p. 149 - 164, 2004.

BARTOSZECK, A. C. de P. e S.; MACHADO, S. do A.; FIGUEIREDO FILHO, A.; OLIVEIRA, E. B. A distribuição diamétrica para bracatingais em diferentes idades, sítios e densidades na Região Metropolitana de Curitiba. Floresta, Curitiba, v. 34, n. 3, p. 305 - 323, 2004.

CALDATO, S. L.; LONGHI, S. J.; FLOSS, P. A. Estrutura populacional de Ocotea porosa (Lauraceae) em uma Floresta Ombrófila Mista, em Caçador (SC). Ciência Florestal, Santa Maria, v. 9, n. 1, p. 89 101, 1999.

CAMPANILI, M.; SCHAFFER, W. B. Mata Attântica: manual de adequação ambiental. Brasília: MMA/SBF, 2010. $96 \mathrm{p}$.

CAPOBIANCO, J. O. R. Mata Atlântica: conceito, abrangência e área original. In: SCHAFFER, W. B.; PROCHNOW, M. (Org.). A Mata Atlântica e você: como preservar, recuperar e se beneficiar da mais ameaçada floresta brasileira. Brasília: APREMAVI, 2002. p. 111 - 124.

CLUTTER, J. L.; FORTSON, J. C.; PLENAAR, L. V.; BRISTER, G. H.; BAILEY, R. L. Timber management: a quantitative approach. New York: John Wiley \& Sons, 1983. 333 p. 
FIGUEIREDO FILHO, A.; DIAS, A. N.; STEPKA, T. F.; SAWCZUK, A. R. Crescimento, mortalidade, ingresso e distribuição diamétrica em Floresta Ombrófila Mista. Floresta, Curitiba, v. 40, n. 4, p. 763 $776,2010$.

INSTITUTO BRASILEIRO DE GEOGRAFIA E ESTATÍSTICA (IBGE). Manuais técnicos em geociências: Manual técnico da vegetação brasileira. n. 1. 2. ed. Rio de Janeiro: IBGE, 2012. 275 p. 2007. $300 \mathrm{p}$.

Manuais técnicos em geociências: Manual técnico de pedologia. n. 4. 2. ed. Rio de Janeiro: IBGE,

LOETSCH, F.; ZÖHRER, F.; HALLER, K. E. Forest Inventory. München: BLV Verlagsgesellschaft, 1973. $469 \mathrm{p}$.

MACHADO, S. A.; BARTOSZEK, A. C. P. S.; OLIVEIRA, E. B. Estudo da estrutura diamétrica para a Araucaria angustifolia em florestas naturais na região Sul do Brasil. Revista Floresta, v. 26, n. 1 - 2, p. 59 - 70, 1996.

MACHADO, S. do A.; AUGUSTYNCZIK, A. L. D.; NASCIMENTO, R. G. M.; TÉO, S. J.; MIGUEL, E. P.; FIGURA, M. A.; SILVA, L. C. R. da. Funções de distribuição diamétrica em um fragmento de Floresta Ombrófila Mista. Ciência Rural, Santa Maria, v. 39, n. 8, p. 2428 - 2434, 2009 a.

MACHADO, S. do A.; AUGUSTYNCZIK, A. L. D.; NASCIMENTO, R. G. M.; FIGURA, M. A.; SILVA, L. C. R. da; MIGUEL, E. P.; TÉO, S. J. Distribuição diamétrica de Araucaria angustifolia (Bert.) O. Ktze. em um fragmento de Floresta Ombrófila Mista. Scientia Agraria, Curitiba, v. 10, n. 2, p. 103 110,2009 b.

MACHADO, S. do A.; SANTOS, A. A. P. do; NASCIMENTO, R. G. M. do; AUGUSTYNCZIK, A. L. D.; ZAMIN, N. T. Modelagem da Distribuição Diamétrica de Quatro Espécies de Lauraceae em um fragmento de Floresta Ombrófila Mista. Revista Ciências Exatas e Naturais, Guarapuava, v. 12, n. 1, p. $91-105,2010$ a.

MACHADO, S. do A.; NASCIMENTO, R. G. M.; AUGUSTYNCZIK, A. L. D.; MIGUEL, E. P.; TÉO, S. J. Distribution of total height, transverse area and individual volume for Araucaria angustifolia (Bert.) O. Kuntze. Cerne, Lavras, v. 16, n. 1, p. 12 - 21, 2010 b.

MEYER, H. A.; RECKNAGEL, A. B.; STEVENSON, D. D.; BARTOO, R. A. Forest management. 2. ed. New York: Ronald Press, 1961. 282 p.

ORELLANA, E. Funções densidade de probabilidade no ajuste da distribuição diamétrica de um fragmento de Floresta Ombrófila Mista. 2009. 122 f. Dissertação (Mestrado em Ciências Florestais) Universidade Estadual do Centro - Oeste, Irati, 2009.

SCHAAF, L. B.; FIGUEIREDO FILHO, A.; GALVÃO, F.; SANQUETTA, C. R. Alteração da estrutura diamétrica de uma Floresta Ombrófila Mista no período entre 1979 e 2000. Revista Árvore, Viçosa, v. 30, n. 2, p. 283 - 295, 2006.

SCOLFORO, J. R. S. Biometria florestal: modelos de crescimento e produção florestal. Lavras: UFLA/FAEPE, 2006. 393 p. 
FLORESTA, Curitiba, PR, v. 45, n. 2, p. 337 - 348, abr. / jun. 2015. 